\title{
Possible Potential Outcomes from COVID-19 Complications on Testes: Lesson from SARS Infection
}

\author{
Ranjha Khan ${ }^{1}$, Tayyaba Naseem², Muhammad Jafar Hussain ${ }^{3}$, Muhammad Amir Hussain ${ }^{4}$ and Saima Shakil \\ Malik $^{5}$ \\ ${ }^{1}$ The First Affiliated Hospital of USTC, Hefei National Laboratory for Physical Sciences at the Microscale, School of Life Sciences, \\ University of Science and Technology of China, Hefei, Anhui, China \\ ${ }^{2}$ University of Management and Technology (UMT), Johar Town, Lahore, Pakistan \\ ${ }^{3}$ Department of Nephrology, Institute of Nephrology, School of Medicine, Shanghai Jiao Tong University, Shanghai, China \\ ${ }^{4}$ Sharif Medical and Dental College, Sharif Medical City Road, Jati Umra, Lahore, Pakistan \\ ${ }^{5}$ Department of Zoology, University of Gujrat, Pakistan
}

\begin{abstract}
Coronavirus infection disease-2019 (COVID-19) gained worldwide fame after deadly outbreak in China and its subsequent spread to many countries. So far, COVID-19 is not fully contained, and new cases are arising on daily bases in various countries. Due to zoonotic nature and human-to-human spread, COVID-19 is considered pandemic with more causalities in developing countries. Full genome analysis revealed its resemblance with severe acute respiratory syndrome (SARS) virus with minor variation in non-structural proteins. Both viruses use the common angiotensin converting enzyme (ACE2) receptor to attach to the epithelial cells of the target organs. Currently, COVID-19 patients are treated with drugs that are used for lungs infections. However, ACE2 has high expression in other human organs such as kidney and testes. Thus, it is assumed that, like SARS, it may have adverse effects on other vital organs, which have dominant expression of ACE2. In testis, SARS affected patients displayed peritubular fibrosis, extensive germ cell disruption, damage of blood-testis barrier integrity and more frequent occurrence of apoptosis. Here, we critically discuss the possible adverse effects of COVID-19 on the testes of patients along with future precautions to overcome the complications of reproductive organs.
\end{abstract}

Key Words: COVID-19; SARS; ACE2, Testes.

How to cite this article: Khan R, Naseem T, Hussain MJ, Hussain MA, Malik SS. Possible Potential Outcomes from COVID-19 Complications on Testes: Lesson from SARS Infection. J Coll Physicians Surg Pak 2020; 30(JCPSPCR):CR118-CR120.

Coronaviruses (CoVs) are complex type of RNA viruses which are classified into four genera, i.e., Alphacoronaviruses $(\alpha \mathrm{CoVs})$, Betacoronaviruses ( $\beta \mathrm{CoVs})$, Deltacoronaviruses ( $\delta \mathrm{CoVs})$ and Gammacoronaviruses (rCoVs). Evolutionary analyses have shown that bats and rodents are the sources of most of the $\beta C$ oVs, which are enveloped, positive sense, single stranded RNA (ssRNA) viruses. The important feature of these viruses is the presence of club-like spikes projecting from the surface. CoVs are commonly associated with acute respiratory infections in human beings, but they also have the ability to infect multiple host species, making them complex pathogens. ${ }^{1}$

Correspondence to: Dr. Muhammad Jafar Hussain, Department of Nephrology, Institute of Nephrology, Shanghai Ruijin Hospital, Shanghai, China

E-mail: hafizaasi19@gmail.com

Received: June 10, 2020; Revised: August 16, 2020;

Accepted: August 22, 2020

DOI: https://doi.org/10.29271/jcpsp.2020.JCPSPCR.CR118
Since December 2019, several cases of pneumonia-like disease of unknown cause emerged in the central city of China, Wuhan. Samples were taken from lower portion of respiratory tract and deep sequence analysis showed a novel virus, which was named as novel CoV 2019 (2019-nCoV), and now recognised as CoV infection disease 2019 (COVID-19) by WHO. It spread widely in China and throughout the globe, and still its outbreak is not contained fully. ${ }^{2}$

Full genome sequence analysis showed that the virus causing COVID-19 belongs to $\beta$ CoV and almost $67 \%$ of whole genome of the virus is represented by first open reading frame (ORF) encoding 16 non-structural proteins (NSPs), while accessory proteins and structural proteins are encoded by remaining ORFs. Evolutionary studies have suggested that 2019-nCoV resembles severe acute respiratory syndrome (SARS)-bat like viruses; however, phylogenetic studies have shown that 2019$\mathrm{nCoV}$ has a little divergent relationship with SARS-CoV and middle-east respiratory syndrome (MERS)-CoV. The common feature between SARS and COVID-19 virus is the same angiotensin converting enzyme 2 (ACE2) receptor that enables these viruses to attach to epithelial cells of the respiratory system. ${ }^{3}$ However, the ACE2 receptors are widely distributed in 
various human tissues; and the impact of COVID-19 on other tissues need to be explored.

In order to find out the future possible effects of COVID-19 on various human body tissues, we searched the ACE2 expression in various tissues of humans and found that its expression is comparatively high ingall bladderand testes (Tablel).

Table l: Expression of ACE2 (RPKM) in differenthumantissues.

\begin{tabular}{|l|c|}
\hline Tissues & Expression (RPKM) \\
\hline Gall Bladder & $32.606 \pm 14.37$ \\
\hline Brain & 0 \\
\hline Heart & $12.309 \pm 10.958$ \\
\hline Small intestine & $93.724 \pm 16.10$ \\
\hline Kidney & $30.81 \pm 17.148$ \\
\hline Liver & $1.294 \pm 0.368$ \\
\hline Lung & 0 \\
\hline Colon & $4.695 \pm 1.662$ \\
\hline Stomach & $1.177 \pm 0.937$ \\
\hline Spleen & 0 \\
\hline Thyroid & $1.392 \pm 0.928$ \\
\hline Testis & $26.895 \pm 8.944$ \\
\hline Duodenum & $69.049 \pm 6.292$ \\
\hline Fat & $2.389 \pm 1.378$ \\
\hline Placenta & $0.675 \pm 0.102$ \\
\hline Prostate & 0 \\
\hline $\begin{array}{l}\text { RPKM: Reads per kilo base per million base mapped reads. } \\
\text { Source: http://www.ncbi.nlm.nih.gov }\end{array}$ & \\
\hline
\end{tabular}

Surprisingly, ACE2 expression is not observed in the lungs where it has most adverse effects; however, the structural protein called spike surface glycoprotein has an important role in binding to receptors on the cells of the host, thus, determining host response. In this short-review, we propose future concerns of COVID-19 on reproductive organs and these concerns are linked to previous published literature of SARS effects on them.

In the previous decade, China faced the deadly outbreak of SARS that infected around 8,000 people with almost 800 deaths. The main clinical manifestations were: common cold, respiratory tract infections, fatigue and diarrhoea. It was zoonotic infection with later human-to-human spread. ACE2 is the most important receptor for SARS and is an important enzyme that regulates cardiovascular and renal functions in humans. It was observed that SARS outbreak was not only limited to the respiratory system, it also adversely affected the other organs including kidney and testes. ${ }^{4}$ The specifical effect of SARS on testes morphology was explored; and it was found that phenotypically different effects were observed on morphology of testes in different patients. Generally, testes displayed peritubular fibrosis, extensive germ cell disruption as well thickened seminiferous epithelial linings. Subsequently, terminal deoxynucleotidyl transferase dUTP nick end labeling (TUNEL) assay revealed that most spermatids undergo frequent apoptosis and some Leydig cells also showed apoptosis, thus, leading to severe reduction of sperm concentration in the lumen of tubules. ${ }^{5}$ Yang et al., reported that testis from COVID-19 patients demonstrated significant seminiferous tubular injury, mild lymphocytic inflammation and reduced Leydig cells, representing testis involvement in COVID-19 viral infection. ${ }^{6} \mathrm{~A}$ recent study found that ACE2 receptor, a target for COVID-19 infection, is expressed in germ cells, Leydig cells, and Sertoli cells in the testis using single-cell RNA sequencing, suggesting that testis is potentially a target for COVID-19 infection. ${ }^{7}$ Recent literature has confirmed that COVID-19 utilises the same cellular receptor, ACE2, as the SARS $\mathrm{COV}$. The S glycoprotein of CoVs binds with ACE2 receptor on human cells' surface leading to its entry into the cells, ${ }^{8}$ and various approaches are in progress to explore and inhibit this binding. ${ }^{9}$ Moreover, it was found that COVID-19 genotype mutated in different patients in China, ${ }^{10}$ emphasizing in-depth investigations of epidemic and virulence.

The blood-testes barrier (BTB) is established by the sertoli cells (SCs), the well-known testicular somatic cells, essential for the nurturing and development of testicular germ cells. This wellestablished BTB is responsible for the protection of germ cells from immunological cells especially lymphocytes and T cells. The BTB is mainly constituted by tight junctions (TJs), ectoplasmic specialisation (ES), and gapjunctions (GJs) between the adjacent SCs in vicinity of basal membrane of seminiferous tubules. ${ }^{10}$ The BTB divides seminiferous epithelium into basal and adluminal compartments. Only limited number of $\mathrm{CD}^{+}$and $\mathrm{CD} 8^{+}$cells are observed in normal individuals that are filtered out from the seminiferous tubules. In contrast to this, testicular histology of patients affected with SARS showed increased numbers of $T$ lymphocytes and macrophages, ranging from $4.49 \%$ to $11.72 \%$, respectively. ${ }^{5}$ These findings suggest that BTB integrity is compromised in SARS patients. Furthermore, IgG immunoreaction was significantly higher in the patient's seminiferous tubules as compared to controls, suggesting immune response as the probable cause of orchitis. Thus, it is inferred that SARS infection is not only limited to lungs, but has adverse effects on other vital organs.

Generally, it is considered that virus infection depends upon the expression and distribution of its target receptors on the organs. Due to higher expression of ACE2 in testes (Table I), it is hypothesised that COVID-19 can affect the fertility of patients similar to SARS. Thus, we have suggested some possible concerns and their follow-up points related to reproductive status of patients.

During the quarantine phase, the clinicians should pay more attention to testicularlesions.

COVID-19 can possibly damage the BTB integrity similar to SARS; thus, clinical follow-up of recovered patients is necessary.

As the COVID-19 can affect the new born children from affected mothers, it is possible that male reproductive system of the baby boy may be infected and may cause infertility.

Infertility is a social disorder having psychological effects on couple, thus recovered COVID-19 patients should pay more attention, if they face inability of producing child in a year of unprotected intercourse, and consult the clinicians. 
The patients may try to freeze their healthy sperms by using assisted reproductive technologies to avoid future complications.

These are some possible points that need to be adopted to protect the fertility of patients. However, so far, no clinical data has been published from the testicular biopsies of COVID-19 patients. Thus, our concerns need to be experimentally verified as all our suggestions are based on the previously published SARS literature.

\section{CONFLICT OF INTEREST:}

The authors declared no conflict of interest.

\section{AUTHORS' CONTRIBUTION:}

RK, TN, SSM: Have collected the data and corelated it with the previously reported literature and done the writeup.

$\mathrm{HMAH}$ : Has analysed the clinical aspects of the data and helped in writeup.

$\mathrm{HMJH}$ : Has purposed, supervised and approved the final version of the manuscript.

\section{REFERENCES}

1. Phan T. Novel coronavirus: From discovery to clinical diagnostics. Infect Genet Evol 2020; 104211. doi.org/ 10.1016/j.meegid.2020.104211.

2. Li Q, Guan X, Wu P, Wang X, Zhou L, Tong Y, et al. Early transmission dynamics in Wuhan, China, of novel coronavirus-infected pneumonia. N Engl J Med 2020; 382(13):1199-1207. doi: 10.1056/NEJMoa2001316.

3. Ge XY, Li JL, Yang XL, Chmura AA, Zhu G, Epstein JH, et al.
Isolation and characterization of a bat SARS-like coronavirus that uses the ACE2 receptor. Nature 2013; 503(7477):535-8. doi: 10.1038/nature12711.

4. He L, Ding $Y$, Zhang Q, Che X, He Y, Shen $H$, et al. Expression of elevated levels of pro-inflammatory cytokines in SARS-CoV-infected ACE2+ cells in SARS patients: Relation to the acute lung injury and pathogenesis of SARS. J Pathol 2006; 210(3):288-97. doi: 10.1002/path.2067.

5. Xu J, Qi L, Chi X, Yang J, Wei X, Gong E, et al. Orchitis: A complication of severe acute respiratory syndrome (SARS). Biol Reprod 2006; 74(2):410-6. doi: 10.1095/ biolreprod.105.044776.

6. Yang $M$, Chen S, Huang B, Zhong JM, Su H, Chen YJ, et al. Pathological findings in the testes of COVID-19 patients: Clinical implications. Eur Urology Focus 2020; 6(5):1124-9. doi: 10.1016/j.euf.2020.05.009.

7. Barton LM, Duval EJ, Stroberg E, Ghosh S, Mukhopadhyay S. Covid-19 autopsies, oklahoma, USA. Am J Clin Pathol 2020; 153(6): 725-33. doi: 10.1093/ajcp/aqaa062.

8. Zhang L, Shen FM, Chen F, Lin Z. Origin and evolution of the 2019 novel coronavirus. Clin Infec Dis 2020; 71(15):882-3. doi: 10.1093/cid/ciaa112.

9. Masood N, Malik SS, Raja MN, Mubarik S, Yu C. Unravelling the epidemiology, geographical distribution, and genomic evolution of potentially lethal coronaviruses (SARS, MERS, and SARS CoV-2). Front Cell Infect Microbiol 2020; 10:499. doi: 10.3389/fcimb.2020.00499.

10. Xiaolu $T$, Changcheng $W$, Xiang $L$, Yuhe $S$, Xinmin $Y$, Xinkai $W$, et al. On the origin and continuing evolution of SARS-CoV-2. Natl Sci Rev 2020; 7(6):1012-23. doi.org/ 10.1093/nsr/nwaa036. 